\title{
A TIME-SAVING INCREMENTAL CYCLE ERGOMETER PROTOCOL TO DETERMINE PEAK OXYGEN CONSUMPTION
}

\author{
J. LAWLER, S. K. POWERS and S. DODD \\ Applied Physiology Laboratory, School of Health, Physical Education, Recreation and Dance, Louisiana State University, USA
}

\section{ABSTRACT}

Previously we have demonstrated that an accelerated arm ergometry testing protocol results in a higher peak oxygen consumption than continuous or discontinuous protocols reported in the literature (Brit.J.Sports Med. 20: 25-26, 1986). The purpose of this investigation was to determine if an accelerated protocol was superior to two commonly used protocols in cycle ergometry. Nine male subjects were tested on three different exercise protocols; a discontinuous test (DT), a continuous test (CT) and a new proposed "jump-max test" (JMT). The CT began at a work rate of $70 \mathrm{~W}$ with the power output (PO) being increased $35 \mathrm{~W} \cdot \mathrm{min}^{-1}$. The DT began at a work rate of $70 \mathrm{~W}$; the work rate was increased by $35 \mathrm{~W}$ every 2 minutes with 2 minutes rest between stages. The JMT began with a 3-minute pretest to determine a PO which elicited a HR of $145 \pm 5$ bpm. After a 2-minute rest, subjects began exercise at the predetermined work rate with the PO being increased 35 W.min-1. Testing sessions were terminated when subjects failed to maintain the desired PO. No significant difference $(p>0.05)$ existed in peak $\mathrm{PO}$ or peak oxygen consumption $\left(\mathrm{VO}_{2}\right)$ between the three protocols. However, JMT protocol did result in a shorter time to exhaustion than the other protocols employed $(P<0.05)$.

Key words: $\dot{\mathrm{V}}_{2}$ max, Cycle ergometer, Maximal exercise, Incremental exercise

\section{INTRODUCTION}

The use of graded cycle ergometry tests to determine maximum oxygen uptake $\left(\mathrm{VO}_{2}\right.$ max) or cycle ergometer peak oxygen uptake (peak $\mathrm{V}_{2}$ ) is common in exercise physiology laboratories around the world. Data obtained from these tests have been used for diagnostic purposes, assessment of cardiopulmonary fitness, and evaluation of training regimens.

Cycle ergometry protocols commonly used to determine peak $\mathrm{VO}_{2}$ may last $\geqslant 15$ minutes ( $\AA$ strand et al, 1959; Fardy et al, 1977; Glassford et al, 1965; Hettinger et al, 1961; Schwade et al, 1977). Tests of this duration may be terminated by a perception of local fatigue, as well as elevated body temperature and greater dehydration, instead of the central or peripheral limits of oxygen consumption (Buchfuhrer et al, 1983; Fardy et al, 1977; McKay and Banister, 1976). This may be especially true for subjects who are not highly aerobically trained (Balke and Ware, 1959). It could be postulated that if the cardiorespiratory capacity can be maximally stressed before local fatigue or a lack of subject commitment limits the work, a higher peak oxygen uptake may be achieved during cycle ergometry. This notion is supported by Buchfuhrer et al (1983) who have suggested that, in order to optimise the measurement of $\dot{\mathrm{VO}}_{2}$ max or peak $\dot{\mathrm{VO}}_{2}$, subjects be given a work rate increment that would allow them to reach their maximum exercise tolerance within 8-17 minutes.

Walker et al (1986) recently demonstrated that a "jumpmax" arm ergometry protocol of short duration produced significantly higher peak $\mathrm{VO}_{2}(P<0.05)$ than two different protocols of longer duration. As peak $\mathrm{VO}_{2}$ measurements in cycle ergometry may also be influenced by the duration of the tests (Fardy et al, 1977), a similar jump-max protocol was proposed for comparison with two established protocols, of continuous and discontinuous nature, respectively. Hence, the purpose of the present

\section{Address for correspondence:}

Dr. S. K. Powers

Applied Physiology Laboratory

School of HPERD

Louisiana State University

Baton Rouge, LA 70803

USA investigation was to determine whether a new accelerated cycle ergometer protocol would elicit a higher peak oxygen consumption than two commonly used protocols of short to moderate duration.

\section{METHODS}

\section{Subjects}

Nine male volunteers, age (mean \pm SD) $27.7 \pm 4.4$ years and weight $71 \pm 8.3 \mathrm{~kg}$ agreed to participate in this study. All subjects were moderately aerobically trained (i.e. running 3-5 times weekly), healthy, non-smoking individuals with no history of pulmonary or cardiovascular disease. Participants were informed of the nature of the study and signed consent forms as prescribed by the Louisiana State University Human Subjects Committee before testing commenced.

\section{Test Protocol}

Subjects reported to the laboratory 4-6 hours postprandial at the same time of the day on three separate occasions and performed a different incremental cycle ergometry test to volitional exhaustion each time. Tests were separated by at least 72 hours. On each day subjects completed one of the three incremental exercise protocols, assigned in counterbalanced order: a discontinuous test (DT), a continuous test (CT), and the new jump-max test (JMT).

The DT utilised a protocol similar to one proposed by Fardy et al (1977). The test began with a 3-minute warm-up at a power output (PO) of $70 \mathrm{~W}(70 \mathrm{rpm})$ followed by a 2minute rèst period. The protocol then required subjects to pedal for 2-minute periods with 2-minutes of rest between incremental stages. Power output was increased by $35 \mathrm{~W}$ per stage and the test was terminated when the subject could no longer maintain the desired PO.

The CT commenced with a 3-minute warm-up at $70 \mathrm{~W}(70$ rpm) with a 2-minute rest period following. Thereafter, subjects worked continuously in a manner similar to that described by Michael and Horvath (1965). Power output was increased by $35 \mathrm{~W} \cdot \mathrm{min}^{-1}$ and the test continued until the desired $P O$ could not be maintained.

The JMT began with a 3-minute pretest designed to determine a PO that would increase the subject's heart rate (HR) to $75 \%$ of the age predicted maximal heart rate $(145 \pm$ 5 beats. $\min ^{-1}$ ) (Walker et al, 1986). The pretest commenced 
at a work rate of $35 \mathrm{~W}(70 \mathrm{rpm})$ with the PO increased $35 \mathrm{~W}$ every 30 seconds until the subject attained the desired target HR, i.e. $145 \pm 5$ beats. in $^{-1}$. Subjects who reached the target HR prior to 3 minutes continued pedalling, but now at $50 \%$ of the determined target power output for the duration of the pretest. A 2-minute recovery period followed the pretest. The JMT then resumed, at the predetermined PO that elicited the target HR, with the PO being increased $35 \mathrm{~W} \cdot \mathrm{min}^{-1}$. The test was terminated when the desired power output could no longer be maintained.

Subjects were encouraged verbally to exercise as long as possible for each test protocol. Each session was determined to be a successful assessment of peak $\dot{\mathrm{vO}}_{2}$ if two out of the three following criteria were satisfied: 1) identification of a plateau of $\mathrm{VO}_{2}$ with an increase $(\leqslant 150 \mathrm{ml}$. $\left.\min ^{-1}\right)$ in $\mathrm{PO} ; 2$ ) a respiratory exchange ratio $(R) \geqslant 1.1$; and 3) a peak heart rate $\pm 10 \%$ of age-predicted maximum (220 - age).

Subjects performed all tests on a Monark cycle ergometer (model 860) which was calibrated weekly during experimentation. Before each test the seat height on the cycle ergometer was adjusted so each leg was in a position of slight flexion $\left(170^{\circ}\right)$ at the lowest point of the downstroke.

Oxygen uptake, carbon dioxide production $\left(\dot{\mathrm{V}} \mathrm{CO}_{2}\right)$ and inspired ventilation were measured minute-by-minute via open-circuit spirometry. Subjects breathed through a Daniel's low resistance non-rebreathing valve $(80 \mathrm{ml}$ dead space) with expired gasses passing through a $45 \mathrm{~cm}$ length of $34 \mathrm{~mm}$ diameter tubing into a 5 I mixing chamber. Gas was sampled from a mixing chamber port and expired fractions monitored by a Beckman OM-11 oxygen analyser and a Beckman LB-2 carbon dioxide analyser (sampling rate $=500 \mathrm{ml} \cdot \mathrm{min}^{-1}$ per analyser). The gas analysers were calibrated immediately before each test began, between each stage of the DT and upon completion of each test using standardised gases that had been assayed using Scholander analysis (1947). Inspired ventilation was measured by a calibrated Parkinson-Cowan CD-4 dry gas meter fitted with a potentiometer.

Analog (0-5 V) output signals from the oxygen and carbon dioxide analysers and the dry gas meter were processed by an 8-bit RADM-16 analog-to-digital converter (100 $\mu \mathrm{sec}$ conversion time). Resultant digital information was then interfaced with an Apple lle microcomputer which provided a hard copy of all calculated variables (i.e. expired ventilation $\left(\dot{\mathrm{V}}_{\mathrm{E}}\right), \dot{\mathrm{V}} \mathrm{O}_{2}, \dot{\mathrm{V}} \mathrm{CO}_{2}$, etc.). Heart rate was monitored during the last $10-\mathrm{sec}$ of each stage in all tests via electrocardiography. Data were tested for significant differences using one-way analysis of variance. When appropriate, a Newman-Keuls exam was used post-hoc. Significance was established at the 0.05 level of confidence.

\section{RESULTS}

During the course of the experiments two subjects failed to meet the established criteria for peak $\dot{\mathrm{V}}_{2}$. These subjects were retested until the criteria were met. Mean ( \pm SEM) values for peak $\dot{V}_{2}$, peak $H R$, peak $P O, \dot{V}_{E}$, respiratory frequency $(f)$ and the respiratory exchange ratio ( $R$ ) during maximal exercise are presented in Table $I$.

No significant difference existed in peak $\mathrm{VO}_{2}$ (absolute or relative) between the three protocols. Additionally, no significant differences existed in peak HR, peak $P O, \dot{V}_{E}, f$, and $R$ among the three exercise protocols. In contrast, total test time for the JMT was significantly shorter than either the CT or DT. Finally, total test time for the CT was significantly shorter than the time required to complete the DT.

TABLE I

Peak values for oxygen uptake, heart rate, power output, expired ventilation, frequency of breathing, respiratory exchange ratio and test time for three different protocols.

\begin{tabular}{|c|c|c|c|}
\hline Variable & Discontinuous Test & Continuous Test & Jump-max Test \\
\hline $\begin{array}{l}\text { Peak VO } \\
\text { (I.min-1) }\end{array}$ & $3.61 \pm 0.22$ & $3.71 \pm 0.18$ & $3.64 \pm 0.18$ \\
\hline $\begin{array}{l}\text { Peak } \dot{V O}_{2} \\
\left(\mathrm{ml} \cdot \mathrm{min}^{-1} \cdot \mathrm{kg}^{-1}\right)\end{array}$ & $51.1 \pm 2.9$ & $52.6 \pm 2.7$ & $51.7 \pm 3.0$ \\
\hline $\begin{array}{l}\text { Peak HR } \\
\text { (beats } \text { min }^{-1} \text { ) }\end{array}$ & $183.0 \pm 3.2$ & $184.0 \pm 2.8$ & $182.0 \pm 3.1$ \\
\hline $\begin{array}{l}\text { Peak PO } \\
\text { (W) }\end{array}$ & $311.0 \pm 15.2$ & $311.0 \pm 14.0$ & $315.0 \pm 14.6$ \\
\hline $\begin{array}{l}V_{E^{1}} \\
\text { (I.min-1, BTPS) }\end{array}$ & $121.7 \pm 4.0$ & $117.2 \pm 4.9$ & $121.8 \pm 5.1$ \\
\hline $\begin{array}{l}f^{1} \\
\text { (breaths. } \min ^{-1} \text { ) }\end{array}$ & $45.0 \pm 6.2$ & $47.0 \pm 4.3$ & $44.0 \pm 2.9$ \\
\hline $\mathbf{R}^{1}$ & $1.17 \pm 0.020$ & $1.20 \pm 0.018$ & $1.24 \pm 0.030$ \\
\hline $\begin{array}{l}\text { Total time } \\
\text { (min) }\end{array}$ & $29.3 \pm 1.54$ & $13.1 \pm 0.14^{* *}$ & $9.1 \pm 0.32^{*}$ \\
\hline
\end{tabular}

$1 \grave{V}_{E}, f$, and $R$ represent measured variables during the last minute of exercise.

2 Total test time represents the total elapsed time from the beginning of exercise until cessation of exercise.

* Significantly shorter than CT or DT, P $<0.05$

* * Significantly shorter than DT, $P<0.05$

\section{DISCUSSION}

These data do not support the hypothesis that the proposed JMT elicits a higher cycle ergometer peak $\dot{\mathrm{VO}}_{2}$ than the CT or DT. The present findings for incremental cycle ergometry are in contrast to a previous report from this laboratory which suggested an accelerated incremental arm ergometry (Walker et al, 1986) protocol yields higher peak $\dot{\mathrm{VO}}_{2}$ values than traditional continuous or discontinuous protocols.

What is the explanation for this apparent discrepancy between arm and leg exercise protocols? At least two possibilities exist. First, it has been argued that arm exercise may be limited by perfusion and the resulting local muscle fatigue (Gleser and Vogel, 1973; Kamon and Pandolf, 1972; Magel et al, 1978; Washburn and Seals, 1983). This perfusion limitation can be viewed as an indirect function of the relatively small muscle mass in arm exercise compared with leg exercise. The result is that during heavy arm exercise, extramuscular pressures may exceed arterial pressures (Gleser and Vogel, 1973; Petrofsky et al, 1981), thus limiting working muscle capillary bed flow. The overall muscle mass involved in cycle ergometry is much greater than that used during arm ergometry ( $\AA$ strand and Saltin, 1961; Fardy et al, 1977) and it seems unlikely that peripheral factors limit peak $\mathrm{VO}_{2}$ during leg exercise (Schwade et al, 1977; Washburn and Seals, 1983). Therefore, it may be that the inherent physiological mechanisms that limit peak $\mathrm{VO}_{2}$ differ between arm and leg exercise and should be considered in the selection of an exercise testing protocol. This hypothesis warrants further investigation.

A second explanation for the divergent findings may be related to subject training differences between studies Subjects studied by Walker et al (1986) were not trained for arm exercise while subjects used in the present experiments were accustomed to regular bouts of aerobic 
leg exercise. Individuals who are untrained for arm work have been shown to demonstrate a lower lactate threshold as well as an increase in rate of lactate release as compared with trained individuals for cycle ergometry (Pendergast et al, 1979). The resultant early disruption of homeostasis may be attenuated for untrained individuals performing incremental arm ergometry using an accelerated incremental protocol such as that used by Walker et al (1986). One would expect to find that the moderately aerobically trained subjects in the present study to have increased oxidative enzyme activity, higher myoglobin concentration, higher mitochondrial density (Holloszy and Booth, 1976), and increased vascular bed capillarisation (Saltin, 1977) in the exercising muscle. The result may be a lowered glycolytic flux at any given work rate and enhanced lactate clearance. Thus, the accelerated cycle ergometry protocol may not have been as advantageous to this subject pool in order to achieve a higher peak $\mathrm{VO}_{2}$. The possible interaction between state of training and test protocol warrants further investigation.

Although the proposed JMT protocol to determine peak oxygen consumption offers a time saving advantage when compared to the total time of test administration involved in the DT and CT (Table I), the JMT presents a disadvantage in that it does not allow for accurate determination of the anaerobic threshold or the ventilatory threshold. Further, the proposed JMT would not seem appropriate for patients with heart or lung disease since this protocol may not provide the slow work rate progression often required for clinical cardiopulmonary assessment (Buchfuhrer et al, 1983).

In any experiment designed to compare exercise protocols for cardiopulmonary assessment, it is essential that the experimental design employ a set of "fix $\mathbf{J}$ criteria" to determine peak $\mathrm{VO}_{2}$ and a highly motivated si jject pool. The present experiments met both of the above criteria. First, all but two of the subjects reached the established criteria for peak $\dot{\mathrm{VO}}_{2}$ on each of the individual tests. The two subjects who failed to meet the established criteria were retested and both obtained the required peak $\mathrm{VO}_{2}$ criteria upon the second test. Secondly, the nine subjects chosen for study were highly motivated individuals. Hence, it seems unlikely that the results obtained in the present experiments were due to a lack of sustained subject commitment.

In summary, these data do not support the notion that the proposed JMT elicits a higher peak $\mathrm{VO}_{2}$ during cycle ergometry than the continuous or discontinuous tests studied. However, the JMT does save time while achieving similar results. Therefore, it appears that the proposed JMT might be particularly useful in studies requiring determina- tion of peak $\mathrm{VO}_{2}$ in large numbers of subjects.

\section{References}

Åstrand, I., Ástrand, P.-O. and Rodahl, K., 1959 "Maximal heart rate during work in older men". Journal of Applied Physiology 14: 562-566.

Astrand, P.-O. and Saltin, B., 1961 "Maximal oxygen uptake and heart rate in various types of muscular activity". Journal of Applied Physiology 16: 977-981.

Balke, B. and Ware, R. W., 1959 "An experimental study of "physical fitness" of Air Force personnel". U.S. Armed Forces Medical Journal 10: 675-688.

Buchfuhrer, M. J., Hansen, J. E., Robinson, T. E., Sue, D. Y., Wasserman, K. and Whipp, B. J., 1983 "Optimising the exercise protocol for cardiopulmonary assessment". Journal of Applied Physiology 55: 1558-1564.

Fardy, P. S., Webb, D. and Hellerstein, H. K., 1977 "Benefits of arm exercise in cardiac rehabilitation". Physician and Sports Medicine, October: 31-41, 1977.

Glassford, R. G., Baycroft, G. H. Y., Sedgwick, A. W. and McNab, R. B., 1965 "Comparison of maximal oxygen uptake value determined by predicted and actual methods". Journal of Applied Physiology 20: 509-513.

Gleser, M. A. and Vogel, J. A., 1973 "Endurance capacity for prolonged exercise on the bicycle ergometer". Journal of Applied Physiology 34: 438-442.

Hettinger, T., Birkhead, N. C., Horvath, S. M., Issekutz, B. and Rodahl, B., 1961 "Assessment of physical work capacity". Journal of Applied Physiology 16: $153-156$.

Holloszy, J. O. and Booth, F. W., 1976 "Biochemical adaptations to endurance exercise in muscle". Annual Reviews in Physiology 38: 273-291.

Kamon, E. and Pandolf, K. B., 1972 "Maximal aerobic power during laddermill climbing, uphill running, and cycling". Journal of Applied Physiology 32: 467-473.

Magel, J. B., McArdle, W. D., Tone, T.and Delio, D. J., 1978 "Metabolic and cardiovascular adjustment to arm training". Journal of Applied Physiology 45: 75-79.

McKay, S. A. and Banister, E. W., 1976 "A comparison of maximum oxygen uptake determination by bicycle ergometry at various pedalling frequencies and by treadmill running at various speeds". European Journal of Applied Physiology 35: 191-200.

Michael, E. D. Jr. and Horvath, S. M., 1965 "Physical work capacity of college women". Journal of Applied Physiology 20: 263-266.

Pendergast, D., Cerretelli, P. and Rennie, D. W., 1979 "Aerobic and glycolytic metabolism in arm exercise". Journal of Applied Physiology 47: 754-760.

Petrofsky, J. S., Phillips, C. A., Sawka, M. W., Hanpeter, D. and Stafford, D., 1981 "Blood flow and metabolism during isometric contractions in cat skeletal muscle". Journal of Applied Physiology 50: 493-502.

Saltin, B., 1977 "The interplay between peripheral and central factors in the adaptive response to exercise and weight training". Annals of the New York Academy of Sciences 301: 224-242.

Scholander, D., 1947 "Analyzer for accurate estimation of respiratory gases in one-half cubic centimeter samples". Journal of Biological Chemistry 21: 1108-1116.

Schwade, J., Blomqvist, G. G., Shapiro, W., 1977 "A comparison of the response to arm and leg work in patients with ischemic heart disease". American Heart Journal 94: 203-208.

Walker, R., Powers, S. K. and Stuart, M. K., 1986 "Peak oxygen uptake in arm ergometry, effects of testing protocol". British Journal of Sports Medicine 20: 25-26.

Washburn, R. A. and Seals, D. R., 1983 "Comparison of continuous and discontinuous protocols for determination of peak oxygen uptake in arm cranking". European Journal of Applied Physiology 51: 3-6.

Withers, R. T., Sherman, W. M., Miller, J. M. and Costill, D. L., 1981 "Specificity of anaerobic threshold in endurance trained cyclists and runners". European Journal of Applied Physiology 47: 93-104.

\section{ERRATA}

Details of two texts reviewed in 21:3 were incorrectly recorded. The correct information is as follows:

21:3, p. 124: W. E. Prentice, Therapeutic Modalitis in sports medicine. Publisher C. V. Mosby, UK agents Blackwell Scientific. Price $\$ 24$.

21:3, p. 129: D. Peterson, G. Lapenskie and A. W. Taylor: The medical aspects of dance. Publisher: Sports Dynamics, London Ontario. Date: 1986.

We apologise for these errors.
In Dr. Lorna Fisher's review in BJSM 21:3 p. 144 a line was inadvertently omitted, which altered the meaning substantially. The first paragraph should therefore read -

This book is well written and presented with clear headings, tables and illustrations. It is also very well referenced. The authors have tried to addreas the problem of non-articular and non-inflammatory soft tissue heumatic disorders. Reference is made to inflammatory conditions when these need to be considered in the differential diagnosis. A very relaxed interpretation of what constituted 'soft tissue' has allowed the inclusion of conditions such as osteochondritis, osteomalacia and osteoporosis. Conversely, virtually no mention is made of metabolic and endocrine causes of soft tissue rheumatic pain.

We apologise for this error.

H. E. Robson 